\title{
Surgical ventricular restoration by means of a new technique to preserve left ventricular compliance: The horseshoe repair
}

Paolo Ferrazzi, MD, FETCS, Michele Triggiani, MD, PhD, Attilio Iacovoni, MD, Samuele Pentiricci, Francesco Vattimo, MD, Pervirgilio Parrella, ScD, and Eugenio Quaini, MD, Bergamo, Italy

The STICH trial will elucidate the results of surgical ventricular restoration (SVR) associated with coronary artery bypass grafting in patients with ischemic dilated cardiomyopathy but, while awaiting these findings, the current literature shows that left ventricular (LV) sphericalization, continued LV remodeling, and recurrent mitral valve (MV) regurgitation may occur after SVR. ${ }^{1}$ One possible explanation for these events is the worsening or occurrence of diastolic dysfunction. ${ }^{2}$ Until March 2005, we performed SVR by an endoventricular circular patch plasty (Dor procedure), ${ }^{3}$ which consists of 2 circular purse-string sutures (Fontan stitch) and a polyester fiber patch that is much stiffer than the infarcted myocardium. In the Dor procedure, the amount of foreign material used and lateral wall involvement by the Fontan stitch may be responsible for worsening $\mathrm{LV}$ diastolic function, as is shown by the increased slope of the end-diastolic pressure/volume relationship (EDPVR). ${ }^{2}$

A number of authors have described modifications of the Dor technique based on the limited use of foreign material in an attempt to prevent the untoward effect of increased LV stiffness, but none of these has gained wide acceptance.

We describe a new no-patch SVR technique that, by avoiding the use of a circular Fontan stitch, should preserve LV compliance while being as effective as the Dor procedure in reducing LV volumes and diameters.

\section{TECHNIQUE AND RESULTS}

The ideal SVR technique should reduce the equatorial diameter without shortening the longitudinal one.

Our technique consists of 2 horseshoe-shaped, semicircular, parallel purse-string sutures (polypropylene 2-0) positioned in the scarred interventricular septum, acting on the LV circumference: one at equatorial level (which is located at the middle of the insertion of the anterior papillary muscle) ${ }^{4}$ and one displaced $1 \mathrm{~cm}$ toward the apex (Figures 1 and 2). The equatorial purse string is tied first to obtain an

From the Cardiovascular Department, Ospedali Riuniti, Bergamo, Italy.

Received for publication Oct 30, 2007; revisions received Dec 22, 2007; accepted for publication Jan 7, 2008.

Address for reprints: Paolo Ferrazzi, MD, FETCS, Cardiovascular Department, Ospedali Riuniti, Largo Barozzi 1, 24128 Bergamo, Italy (E-mail: pferrazzi@ ospedaliriuniti.bergamo.it).

J Thorac Cardiovasc Surg 2008;136:1382-3

$0022-5223 / \$ 34.00$

Copyright (c) 2008 by The American Association for Thoracic Surgery doi:10.1016/j.jtcvs.2008.01.051

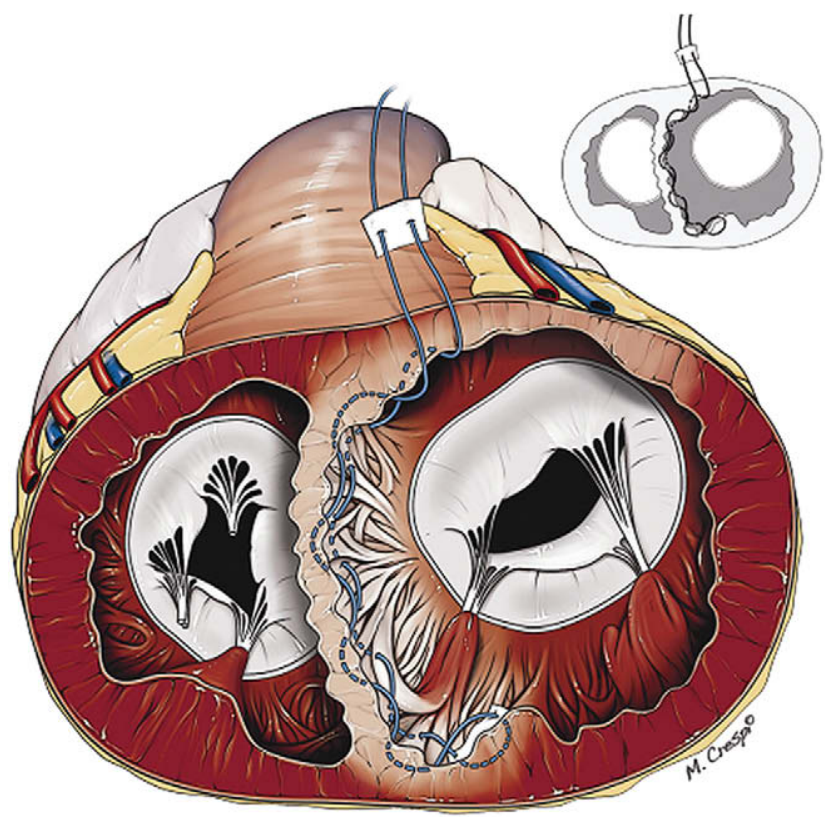

FIGURE 1. Drawing of the cross section of the horseshoe-shaped equatorial semicircular purse-string suture positioned in the scarred interventricular septum.

elliptic shape, and then the second purse string is tied to reshape the new apex. In case of very enlarged LV cavity, 3 (or 4) horseshoe purse-string sutures can be necessary. The ventriculotomy is closed without interposition of a patch

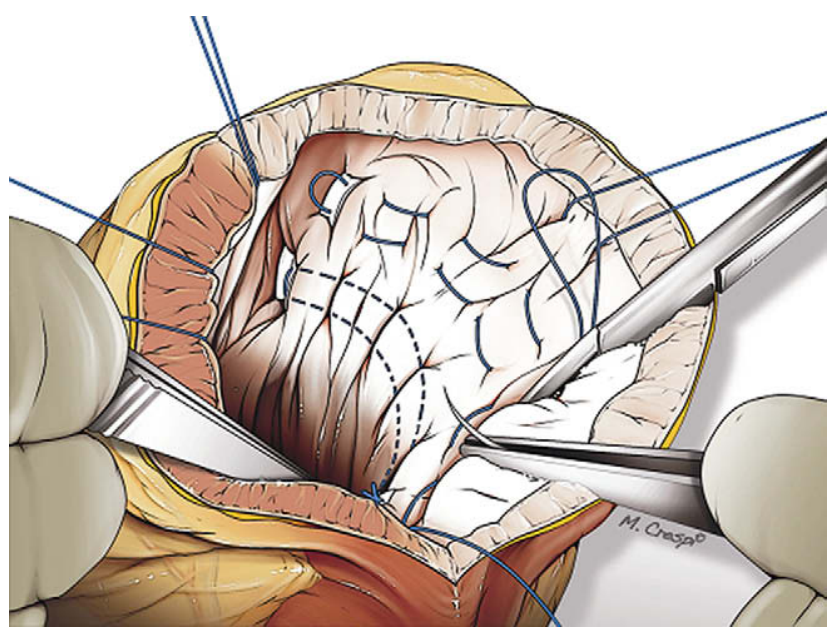

FIGURE 2. Drawing of the surgeon's view of the 2 semicircular pursestring sutures as they appear through the ventriculotomy. 
TABLE 1. Preoperative and follow-up data

\begin{tabular}{lccr}
\hline & Preoperative & Follow-up (mean 6.9 mo) & P value \\
\hline NYHA class & $2.7 \pm 0.8$ & $1.3 \pm 0.5$ & .002 \\
LVEF $(\%)$ & $25 \pm 4.9$ & $45.1 \pm 10.1$ & .001 \\
LVEDV $(\mathrm{mL})$ & $242.4 \pm 60.3$ & $140.9 \pm 32.2$ & $<.001$ \\
LVESV $(\mathrm{mL})$ & $164.8 \pm 58.7$ & $75.0 \pm 23.6$ & .008 \\
LVEDTD $(\mathrm{mm})$ & $64.3 \pm 7.9$ & $57.3 \pm 6.5$ & .004 \\
LVEDLD $(\mathrm{mm})$ & $99.5 \pm 13.4$ & $84.1 \pm 11.6$ & .002 \\
LVEQUD $(\mathrm{mm})$ & $64.1 \pm 5.5$ & $50.3 \pm 7.7$ & $<.001$ \\
EI & $0.73 \pm 0.10$ & $0.76 \pm 0.05$ & .1 \\
\hline
\end{tabular}

NYHA, New York Heart Association; $L V E F$, left ventricular ejection fraction; $L V E D V$, left ventricular end-diastolic volume; $L V E S V$, left ventricular end-systolic volume; LVEDTD, left ventricular end-diastolic transverse diameter; $L V E D L D$, left ventricular end-diastolic longitudinal diameter; $L V E Q U D$, left ventricular equatorial diameter; $E I$, left ventricular diastolic eccentricity index.

or reinforcing felt strips. To avoid shortening the long axis, if the LV longitudinal diameter is $<90 \mathrm{~mm}$, we perform a linear mattress suture reinforced by a running suture (more usual in akinetic LV). If the LV longitudinal diameter is $\geq 90 \mathrm{~mm}$, we use an "overcoat" suture, described by Guilmet in $1984^{5}$ (more usual in dyskinetic LV). We apply this technique in all patients with anteroseptal myocardial infarction, regardless of LV dimensions.

Between April 2005 and November 2006, 15 severely symptomatic (5/15 New York Heart Association class IV, $2 / 5$ under inotropic support) consecutive male patients, aged $61.7 \pm 6.7$ years, with a history of a previous large anteroseptal myocardial infarction underwent the horseshoe repair (Table 1). The surgical protocol consisted of the insertion of a prophylactic intra-aortic balloon pump, induction of normothermic blood cardioplegia, complete myocardial revascularization, MV repair (for grade $\geq 2$ regurgitation), and the placement of a permanent LV epicardial lead (in case of dyssynchrony).

Eight patients (53.3\%) underwent MV annuloplasty. A permanent LV lead was implanted in 5 patients $(33.3 \%)$, and an early postoperative device was implanted in 2 $(13.3 \%)$. The ventriculotomy was closed by means of linear suture in 10 cases $(66.7 \%)$ and by means of an overcoat suture in $5(33.3 \%)$. No in-hospital mortality occurred. After a mean follow-up of 6.9 months, 14 patients $(93.3 \%)$ were still alive; one patient died of pneumonia 95 days after surgery.
We evaluated pressure-volume loops in 2 patients before and directly after cardiopulmonary bypass as previously described $^{2}$ and found a decrease in the EDPVR slope.

\section{DISCUSSION}

The aim of the horseshoe technique is to spare the LV lateral wall to preserve systodiastolic expansions of the viable lateral myocardial wall, which is frequently normally contractile in patients with a previous anteroseptal infarction. This repair shortens LV equatorial diameter and the distance between the lateral wall and septum, preserving lateral contractility.

Like other SVR techniques, the horseshoe repair improves symptoms and LV ejection fraction by reducing LV volumes and diameters. However, our SVR technique also restores LV geometry, as suggested by the trend toward an improved EI, not reported before, ${ }^{6}$ and the significant shortening of the LV equatorial diameter, which identifies a subgroup of patients with a particularly poor prognosis. ${ }^{4}$ Moreover, the favorable change in EDPVR may indicate that the horseshoe repair leads to less impaired passive diastolic function.

Further studies are necessary to assess the long-term stability of this type of repair, particularly in terms of diastolic function.

\section{References}

1. Di Donato M, Sabatier M, Dor V, Gensini GF, Toso A, Maioli M, Stanley AW, et al. Effects of the Dor procedure on left ventricular dimension and shape and geometric correlates of mitral regurgitation one year after surgery. $J$ Thorac Cardiovasc Surg. 2001;121:91-6.

2. Tulner SAF, Steendijk P, Klautz RJM, Bax JJ, Schalij MJ, van der Wall EE, et al. Surgical ventricular restoration in patients with ischemic dilated cardiomyopathy: evaluation of systolic and diastolic ventricular function, wall stress, dyssynchrony, and mechanical efficiency by pressure-volume loops. J Thorac Cardiovasc Surg. 2006;132:610-20.

3. Dor V. Left ventricular aneurysms: the endoventricular circular patch plasty. Semin Thorac Cardiovasc Surg. 1997;9:123-30.

4. Ferrazzi P, Matteucci MLS, Merlo M, Iacovoni A, Rescigno G, Bottai M, et al. Surgical ventricular reverse remodeling in severe ischemic dilated cardiomyopathy: the relevance of the left ventricular equator as a prognostic factor. $J$ Thorac Cardiovasc Surg. 2006;131:357-63.

5. Guilmet D, Popoff G, Dubois C, Tawil N, Bachet J, et al. A new surgical technique for the left ventricular aneurysm: the overcoat aneurysmoplasty. Preliminary results. 11 cases. Arch Mal Coeur Vaiss. 1984;77:953-8.

6. Cirillo M, Amaducci A, Brunelli F, Dalla Tomba M, Parrella P, Tasca G, et al. Determinants of postinfarction remodeling affect outcome and left ventricular geometry after surgical treatment of ischemic cardiomyopathy. $J$ Thorac Cardiovasc Surg. 2004;127:1648-56. 